\title{
Tetrahedral Framework Nucleic Acids loading Ampicillin improve the Drug Susceptibility against Methicillin-resistant staphylococcus aureus
}

Yue Sun ${ }^{1 \dagger}$, Songhang Li ${ }^{1 \dagger}$, Yuxin Zhang ${ }^{1}$, Qirong Li $^{1}$, Xueping Xie ${ }^{1}$, Dan Zhao ${ }^{1}$, Taoran Tian ${ }^{1}$, Sirong Shi ${ }^{1}$, Lingxian Meng ${ }^{1}$, Yunfeng Lin ${ }^{1,2 *}$

1. State Key Laboratory of Oral Diseases, National Clinical Research Centre for Oral

Diseases, West China Hospital of Stomatology, Sichuan University, Chengdu,

610041, China

2. College of Biomedical Engineering, Sichuan University, Chengdu, 610041, China

† Yue Sun and Songhang Li contributed equally to the work.

*Corresponding Author: Yunfeng Lin

Tel/Fax: 86-28-85503487

Email: yunfenglin@scu.edu.cn

ORCID

Yunfeng Lin: 0000-0003-1224-6561

Yue Sun: 0000-0002-5758-1833 


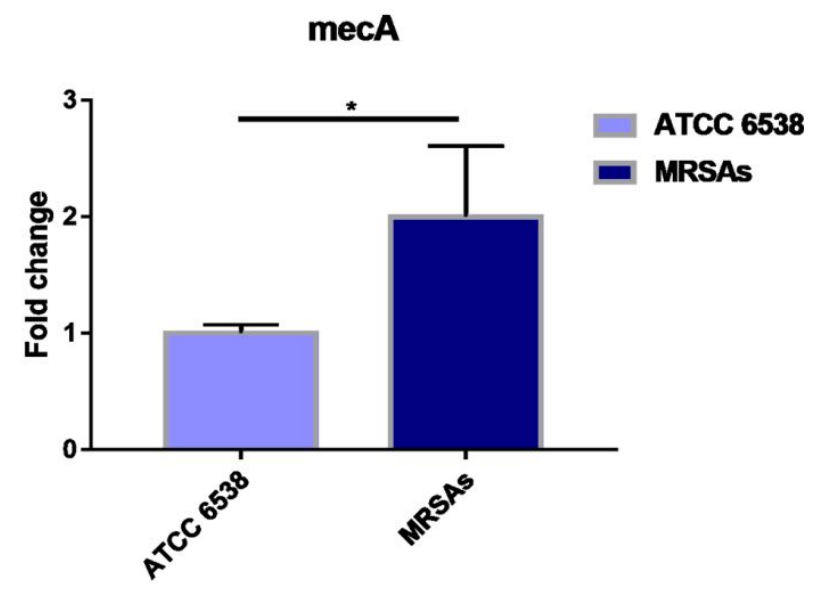

Figure S1. MRSA in experiment is a clinical isolate, and its expression of $m e c A$ gene is different from ATCC 6538. It was proved that MRSA in experiment to be methicillinresistant Staphylococcus aureus strain. Data are mean $\pm \operatorname{SD}(n=3)$. Statistical analysis: ${ }^{*} \mathrm{p}<0.05, * * \mathrm{p}<0.01, * * * \mathrm{p}<0.001$

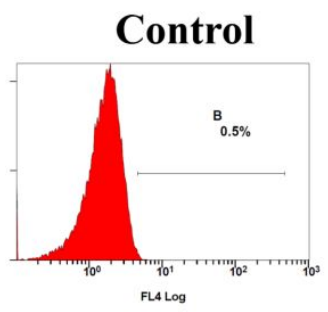

tFNAs

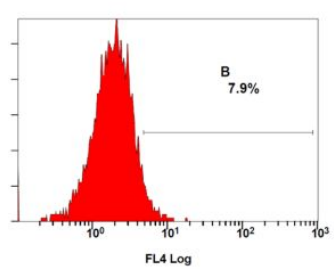

SSDNA

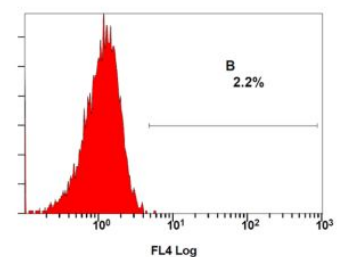

tFNAs-Ampicillin

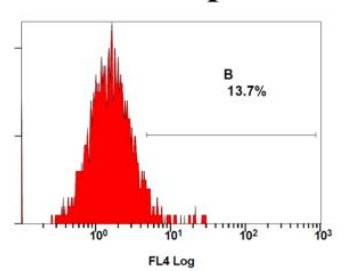

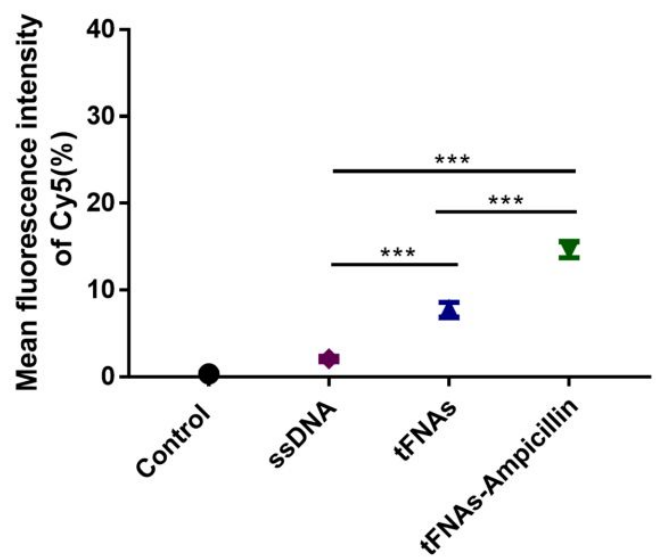


Figure S2. Affinity of tFNAs to $M R S A$ in $6 \mathrm{~h}$. Flow cytometry analysis of the uptake rates of MRSA incubated with tFNAs and tFNAs-Ampicillin for 6h. Quantitative analysis of uptake rates of ssDNA, tFNAs, tFNAs-Ampicillin in flow cytometry analysis. Data are mean $\pm \mathrm{SD}(\mathrm{n}=3)$. Statistical analysis: $* \mathrm{p}<0.05, * * \mathrm{p}<0.01,{ }^{* * *} \mathrm{p}$ $<0.001$.
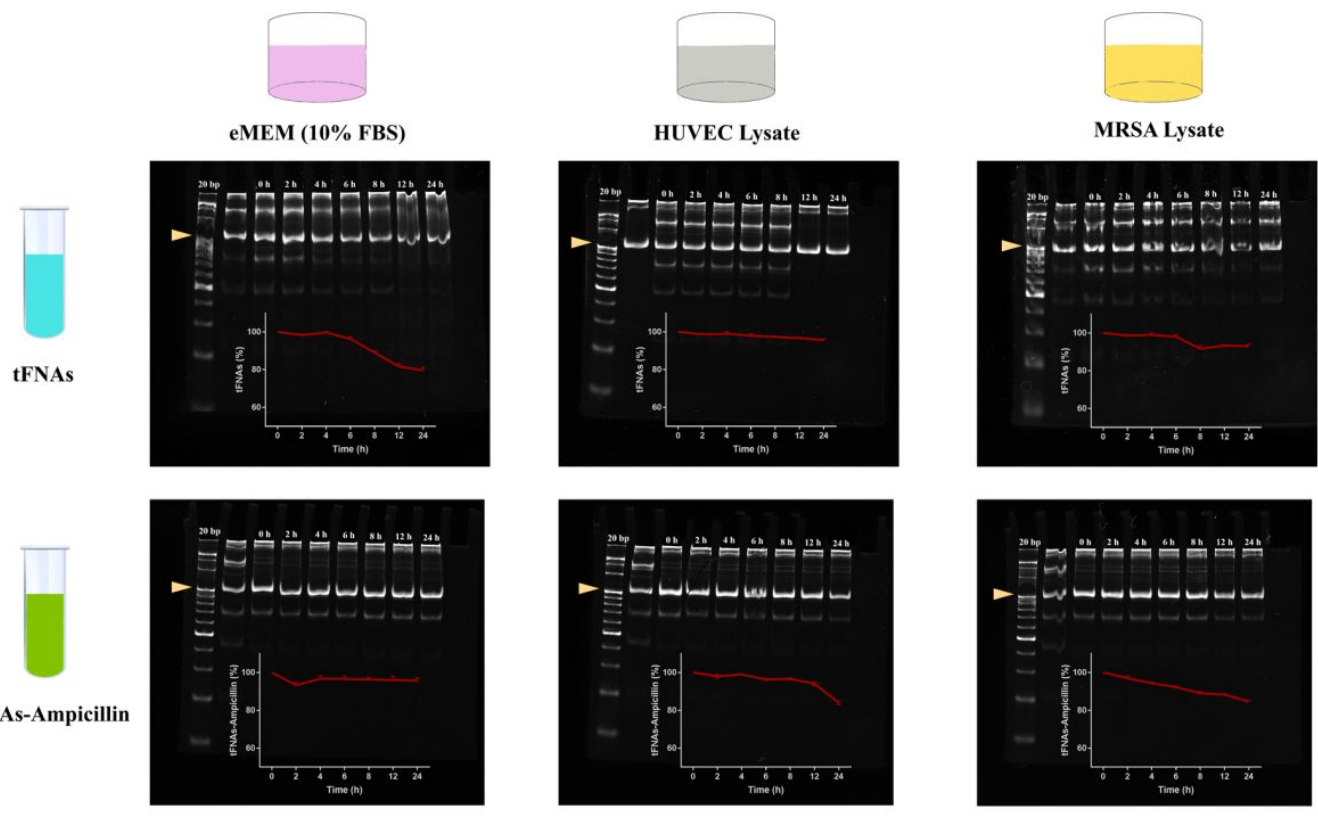

Figure S3. Electrophoretic analysis of the stability of tFNAs and tFNAs-Ampicillin nanostructure. tFNAs and tFNAs-Ampicillin were incubated in eMEM medium (with 10\% FBS), HUVEC lysate (Protein concentration:500 ug/ml) and MRSA 18908 lysate (Protein concentration: Protein concentration:500 ug/ml) at $37{ }^{\circ} \mathrm{C}$ for $0-24 \mathrm{~h}$ and then analyzed with polyacrylamide gel electrophoresis (PAGE). 